\title{
Quantum gates implementations in the separated ion-traps by fast laser pulses
}

\author{
ZHANG Miao $^{1}$ and WEI Lian-Fu*1,2,3 \\ ${ }^{1}$ Quantum Optoelectronics Laboratory, School of Physics and Technology, \\ Southwest Jiaotong University, Chengdu 610031, China \\ ${ }^{2}$ State Key Laboratory of Optoelectronic Materials and Technologies, \\ School of Physics and Engineering, Sun Yat-sen University, Guangzhou 510275, China \\ ${ }^{3}$ State Key Laboratory of Functional Materials for Informatics, \\ Shanghai Institute of Microsystem and Information Technology, \\ Chinese Academy of Sciences, Shanghai 200050, China
}

(Dated: May 29, 2022)

\begin{abstract}
An approach is proposed to implement the universal quantum gates between the ions confined individually in the separated traps. Instead of the typical adiabatic operations, performed for manipulating the ion-ion coupling, here the switchable couplings between ions are implemented non-adiabatically by using the fast laser pulses. Consequently, the desirable quantum gates between the ions could be implemented by using only a series of laser pulses. The proposal may be conveniently generalized to the quantum computation with the scalable ion-traps.
\end{abstract}

PACS numbers: 03.67.Lx, 42.50.Dv, 37.10.Ty

\footnotetext{
*weilianf@mail.sysu.edu.cn; weilianfu@gmail.com
} 
Among the proposed physical systems for building the future quantum computer [1], the system of trapped ions [2-4] is currently one of the most hopeful candidates. Indeed, the entanglement up to fourteen trapped ions has been experimentally realized [5]. In the usual implementations a string of ions are trapped in a single trap, and their center-of-mass mode (CM) acts as the data bus to couple the qubits encoded by the long-lived electronic levels [4]. However, it should be a challenge to trap more ions in a single well and then coherently manipulate them precisely, as it is difficult to obtain the reasonable strong-radial-confinements to form a linear ion-string [4]. Furthermore, the speed of the laser-induced sideband excitation should be significantly reduced with the larger mass of ion-chain.

Alternatively, the scheme of the segmented ion-traps could be an more practical way to implement the large-scale quantum computing network [6-8]. This is because that the ions can be relatively-easily confined in the separated potential wells, and their couplings can be realized by transporting the ions to any selected interaction regions. Specially, if the separate wells are sufficiently close, then the trapped ions can be coupled directly via their Coulomb interaction, and the ion transporting is avoided. Interestingly, such an idea has been demonstrated in recent experiments [9, 10], wherein two ions are confined in two potential wells separated by $40 \mu \mathrm{m}$ [9] (or $54 \mu \mathrm{m}[10]$ ) and the ion-ion coupling is achieved up to $g \approx 10 \mathrm{kHz}$ (or $g \approx 7 \mathrm{kHz}$ ).

In a recent work [11] we have proposed an approach to generate the universal quantum gates between the two separately trapped ions. That idea is based on the experiments [9, 10]: the coupling between the ions was manipulated by controlling the potential wells (i.e., the voltages on the DC electrodes) to adiabatically tune ions' external vibrations into or out of resonance. However, the adiabatic conditions limit the speed of the quantum gates implementing. Also, the exactly-controlling of the potential wells, by adjusting the voltages applied on the DC electrodes, should be very complex for the large-scale ion-traps.

In this letter we propose an improved approach to implement the universal quantum gates between the separately trapped ions. Instead of the previous adiabatic operations applied on the potential wells [9-11], here we use the laser beams to drive one of the trapped ions for controlling its interaction with another ion, let them be resonance or large-detuning. With the present switchable couplings the desirable coherent operations, e.g., the quantum gates, between the separately trapped ions could be performed conveniently by using a series of laser pulses.

We consider the same experimental configuration (shown in Fig. 1) as that in the previous works [9-11], i.e., two ions trapped individually in two potential wells separated by a distance $d$. 


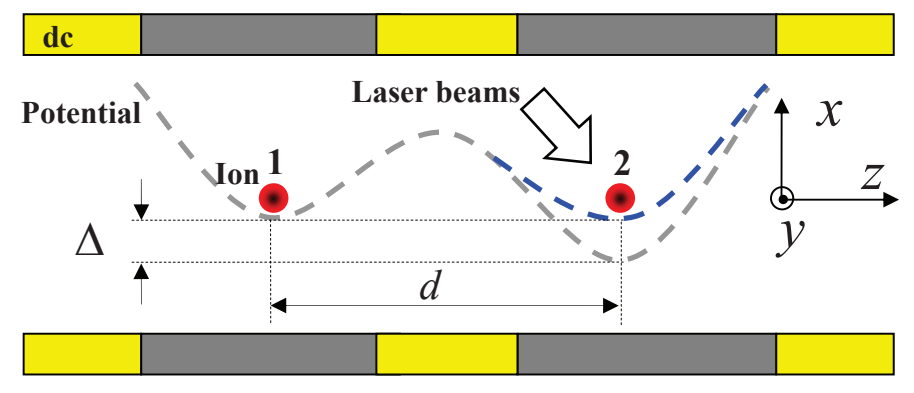

FIG. 1: (Color online) Sketch for implementing switchable coupling between two ions trapped individually in two potential wells (separated by the distance $d$ ). The ions are trapped initially in the large-detuning regime, i.e., they are decoupled at the beginning, then a laser pulse (applied to the ion 2) makes $\Delta=0$ for generating a resonant coupling.

The ion trap provides a pseudopotential such that the ions' oscillating frequencies along the axial direction (i.e., $z$ axis in the Fig. 1) is much smaller than those along the radial directions (i.e., the $x, y$ axis). As a consequence, only the quantized vibrational motion along the axial direction is considered, and the Hamiltonian describing the oscillating ions reads [9-11]

$$
\hat{H}_{\mathrm{ex}}=\sum_{j=1}^{2} \hbar \nu_{j}\left(\hat{a}_{j}^{\dagger} \hat{a}_{j}+\frac{1}{2}\right)+\hat{V}_{\mathrm{ii}}
$$

with

$$
\hat{V}_{\mathrm{ii}}=K\left[\sum_{j=1}^{2} \frac{(-1)^{j} \xi_{j}}{d} \hat{a}_{j}+\frac{\xi_{j}^{2}}{d^{2}}\left(\hat{a}_{j}^{2}+\hat{a}_{j} \hat{a}_{j}^{\dagger}\right)-\frac{2 \xi_{1} \xi_{2}}{d^{2}}\left(\hat{a}_{1} \hat{a}_{2}+\hat{a}_{1} \hat{a}_{2}^{\dagger}\right)\right]+\text { H.c. }
$$

being the ion-ion Coulomb interaction. Where, $\hat{a}_{j}^{\dagger}$ and $\hat{a}_{j}$ are the bosonic creation and annihilation operators of the vibration of frequency $\nu_{j}$. The parameters $K$ and $\xi_{j}$ are defined as $K=q_{1} q_{2} /\left(4 \pi \epsilon_{0} d\right)$ and $\xi_{j}=\sqrt{\hbar /\left(2 M_{j} \nu_{j}\right)}$, with $M_{j}$ and $q_{j}$ being the mass and charge of the ion, respectively.

For realizing a switchable coupling between the two trapped ions, without changing the eigenfrequencies $v_{j}$ of the ions, we apply the laser beams to one of the ions, e.g., the ion 2 . The Hamiltonian describing the laser-driving ion takes the well-known form [12]

$$
\hat{H}_{\mathrm{L}}=\frac{\hbar \omega_{a}}{2} \hat{\sigma}_{z}+\hbar \Omega_{0}\left(\hat{\sigma}_{+}+\hat{\sigma}_{-}\right)\left[e^{i \eta\left(\hat{a}_{2}+\hat{a}_{2}^{\dagger}\right)-i \omega_{l} t-i \phi_{l}}+\text { H.c. }\right] .
$$

Here, $\hat{\sigma}_{z}=|e\rangle\langle e|-| g\rangle\left\langle g\left|, \hat{\sigma}_{+}=\right| e\right\rangle\langle g|$, and $\hat{\sigma}_{-}=|g\rangle\langle e|$ are the Pauli operators of the ion's two internal states $|g\rangle$ and $|e\rangle$ (with the transition frequency $\omega_{a}$ between them); $\Omega_{0}$ is the Rabi frequency describing the strength of the coupling between the applied lasers and the ion; $\eta$ is 
the Lamb-Dicke (LD) parameter describing the strength of the coupling between the external and internal states of the ion; and $\omega_{l}$ and $\phi_{l}$ are the effective frequency and initial phase of the applied laser fields, respectively. If the LD parameter $\eta$ is sufficiently small, then the Hamiltonian in Eq. (3) can be approximated to

$$
\hat{H}_{\mathrm{L}}^{\prime}=\frac{\hbar \omega_{a}}{2} \hat{\sigma}_{z}+\hbar \Omega_{0}\left(\hat{\sigma}_{+}+\hat{\sigma}_{-}\right)\left[\left(1+i \eta\left(\hat{a}_{2}+\hat{a}_{2}^{\dagger}\right)-\frac{\eta^{2}}{2}\left(\hat{a}_{2}+\hat{a}_{2}^{\dagger}\right)^{2}\right) e^{-i \omega_{l} t-i \phi_{l}}+\text { H.c. }\right]
$$

by neglected the higher-order terms $O\left(\eta^{3}\right)[13]$.

Consequently, the total Hamiltonian of the present two-ion system $\hat{H}=\hat{H}_{\mathrm{ex}}+\hat{H}_{\mathrm{L}}^{\prime}$ can be effectively approximated as

$$
\hat{H}^{\prime}=\frac{\hbar \delta_{\text {in }}}{2} \hat{\sigma}_{z}+\hbar \tilde{\Omega} \hat{F}+\hbar\left(\delta_{\text {ex }}-\Omega \hat{F}\right) \hat{a}_{2}^{\dagger} \hat{a}_{2}-\hbar g\left(\hat{a}_{1} \hat{a}_{2}^{\dagger}+\hat{a}_{1}^{\dagger} \hat{a}_{2}\right)
$$

in the rotating framework defined by the unitary operator $\hat{U}_{1}=\exp \left[-i t \omega_{l} \sigma_{z} / 2-i t \sum_{j=1}^{2}\left(\nu_{1} \hat{a}_{j}^{\dagger} \hat{a}_{j}+\right.\right.$ $\left.\left.\nu_{j} / 2\right)\right]$. Above, $\hat{F}=\exp \left(-i \phi_{l}\right) \hat{\sigma}_{+}+\exp \left(i \phi_{l}\right) \hat{\sigma}_{-}, \delta_{\text {ex }}=\nu_{2}-\nu_{1}, \delta_{\text {in }}=\omega_{a}-\omega_{l}, \tilde{\Omega}=1-\eta^{2} / 2$, $\Omega=\Omega_{0} \eta^{2}$, and $g=2 K \xi_{1} \xi_{2} / d^{2}$. Suppose further that the ion is driven resonantly, i.e., $\delta_{\text {in }}=0$, then the Hamiltonian in Eq. (5) reduces to

$$
\hat{H}^{\prime \prime}=\hbar\left(\delta_{\mathrm{ex}}-\Omega \hat{F}\right) \hat{a}_{2}^{\dagger} \hat{a}_{2}-\hbar g\left(\hat{a}_{1} \hat{a}_{2}^{\dagger}+\hat{a}_{1}^{\dagger} \hat{a}_{2}\right)
$$

in another rotating framework defined by the unitary operator $\hat{U}_{2}=\exp (-i t \tilde{\Omega} \hat{F})$. Specially, if the effective phase of the laser beams is set as $\phi_{l}=0$ and the internal state of the ion is prepared in the eigenstate $|\varphi\rangle=(|g\rangle+|e\rangle) / \sqrt{2}$ of the operator $\hat{F}$, then the Hamiltonian in Eq. (6) reduces to

$$
\hat{H}_{\mathrm{eff}}=\hbar \Delta \hat{a}_{2}^{\dagger} \hat{a}_{2}-\hbar g\left(\hat{a}_{1} \hat{a}_{2}^{\dagger}+\hat{a}_{1}^{\dagger} \hat{a}_{2}\right)
$$

with $\Delta=\delta_{\mathrm{ex}}-\Omega$ being the laser-induced controllable detuning between the vibrations. In the previous works [9-11] switchable couplings between the ions were implemented by adiabatically manipulating the vibrational frequencies $\nu_{j}$ (i.e., $\delta_{\mathrm{ex}}$ ). Here, we fixed $\nu_{j}$ (and thus $\delta_{\mathrm{ex}}$ is unchanged) but just adjust the laser-induced effective Rabi frequency $\Omega$ to manipulate $\Delta$. Since $\Omega$ is relativelyeasily controlled by the applied laser and the adiabatic condition is unnecessary to be satisfied, the laser-induced ion-ion coupling/decoupling may be relatively-fast implemented.

The effective Hamiltonian in Eq. (7) yields the dynamics for the bosonic modes

$$
\begin{gathered}
|0\rangle_{1}|1\rangle_{2} \longrightarrow \alpha|0\rangle_{1}|1\rangle_{2}+\beta|1\rangle_{1}|0\rangle_{2} \\
|1\rangle_{1}|0\rangle_{2} \longrightarrow \alpha|1\rangle_{1}|0\rangle_{2}+\beta|0\rangle_{1}|1\rangle_{2}
\end{gathered}
$$




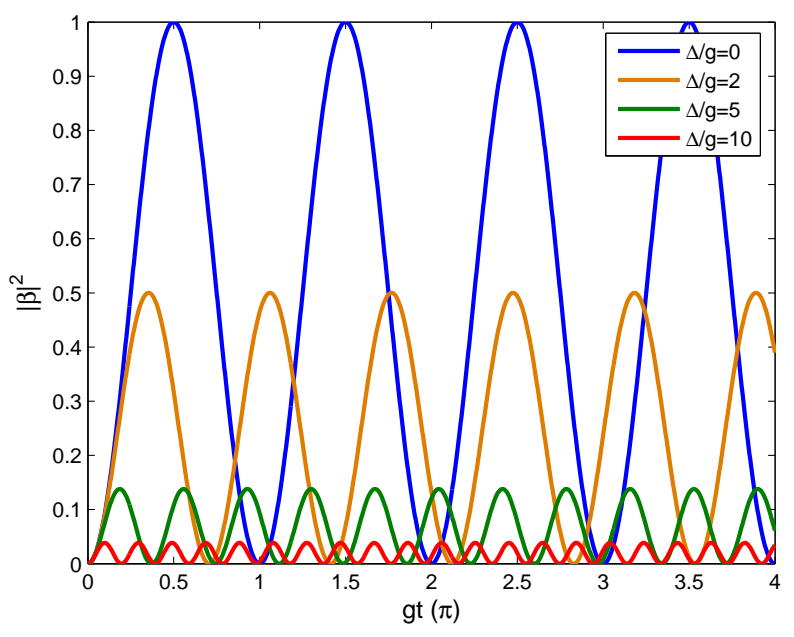

FIG. 2: (Color online) Occupancies $|\beta|^{2}$ in Eq. (8) versus $g t$ for different detunings: $\Delta / g=0, \Delta / g=2$, $\Delta / g=5$, and $\Delta / g=10$. The results indicate that the probability, of the SWAP between the vibrational quanta of the two ions, enhances with the decrease of detuning $\Delta$.

with

$$
\begin{gathered}
\alpha=\left(\frac{1}{2}-\frac{\Delta}{4 c}\right) e^{i(c-\Delta / 2) t}+\left(\frac{1}{2}+\frac{\Delta}{4 c}\right) e^{-i(c+\Delta / 2) t}, \\
\beta=\frac{g}{c} e^{i(\pi-\Delta t) / 2} \sin (c t),
\end{gathered}
$$

and $c=\sqrt{(\Delta / 2)^{2}+g^{2}}$. Above, only two lowest-energy vibrational states of each ion are considered and thus the dynamics is limited within the invariant subspace: $\left\{|0\rangle_{1}|1\rangle_{2},|1\rangle_{1}|0\rangle_{2}\right\}$, with $|0\rangle_{j}$ and $|1\rangle_{j}$ being the ground- and first-excitation states of the $j$ th ion's vibration. The complex parameters $\alpha$ and $\beta$ satisfy the normalized condition $|\alpha|^{2}+|\beta|^{2}=1$, where $|\beta|^{2}$ is just the probability of the SWAP between the vibrational quanta of the ions. Fig. 2 shows how the occupancy $|\beta|^{2}$ changes with $g t$ for different $\Delta$, i.e., $\Delta=0,2 g, 5 g$, and $10 g$, respectively. When $t \sqrt{(\Delta / 2)^{2}+g^{2}}=n \pi / 2$ with $n=0,1,2, \ldots$, the value of $|\beta|^{2}$ reaches its maximum (amplitude), the maximum probability of the desirable SWAP. It is seen that such an amplitude enhances with the decrease of detuning $\Delta$. Therefore, one can turn on or off the coupling between the ionic vibrations by setting $\Delta \approx 0$ or $\Delta \gg g$, correspondingly. Specifically, such a switchable coupling can be implemented as: Initially, the two ions are trapped in separate wells with the detuning $\Delta=\delta_{\text {ex }} \gg g$, i.e., their vibrations are decoupled (shown, e.g., in Fig. 1). Then, a laser pulse with the effective Rabi frequency $\Omega=\delta_{\text {ex }}$ is applied to one of the two ions for realizing the resonant 
coupling (i.e., $\Delta=0$ ). The induced evolution reads

$$
\hat{U}_{1,2}(g t)=\left(\begin{array}{cc}
0 & \cos (g t) \\
i \sin (g t) & 0
\end{array}\right) .
$$

After this laser driving the vibrations of the ions return to their initial decoupled case.

In the above analysis we have assumed $g / \gamma \gg 1$ and neglected the dissipation (with the rate $\gamma$ ) from the environment. Based on the experiments [9, 10], we consider that two ${ }^{40} \mathrm{Ca}^{+}$ions are trapped in different potential wells (separated by the distance $d=40 \mu \mathrm{m}$ ), and their vibrational frequencies are set as $\nu_{1}=5 \mathrm{MHz}$ and $\nu_{2}=5.1 \mathrm{MHz}$. In this case, $g \approx 11 \mathrm{kHz}$ and $\delta_{\mathrm{ex}}=$ $\nu_{2}-\nu_{1}=0.1 \mathrm{MHz}$, which means that the two ions are effectively decoupled from each other. Also, the typical decoherent rate of the ionic vibration is $\gamma_{\mathrm{ex}} \sim 1 \mathrm{kHz}[9,10]$, and the selected electronic levels, $|g\rangle=\left|\mathrm{S}_{1 / 2}\right\rangle$ and $|e\rangle=\left|\mathrm{D}_{5 / 2}\right\rangle$, are long-lived with the decoherent rate $\gamma_{\text {in }} \sim$ $1 \mathrm{~Hz}[14,15]$. Therefore, the condition $g / \gamma \gg 1$ required in our proposal is satisfied. We consider the quadrupole transition (at $729 \mathrm{~nm}$ ) between $\left|S_{1 / 2}\right\rangle$ and $\left|D_{5 / 2}\right\rangle$ [16]. Consequently, the LD parameter is calculated as $\eta \approx 0.1$, working within the LD regime. Experimentally, a laser beam of power $P=140 \mathrm{~mW}$ can generate a quadrupole transition between $\left|\mathrm{S}_{1 / 2}\right\rangle$ and $\left|\mathrm{D}_{5 / 2}\right\rangle$ with Rabi frequency $\Omega_{0}=1.5 \mathrm{MHz}$ [17]. This indicates that the desirable effective Rabi frequency $\Omega=\Omega_{0} \eta^{2}=\delta_{\mathrm{ex}} \approx 0.1 \mathrm{MHz}$ [for implementing the operation in Eq. (11)] could be experimentally realized by applying the laser beam with proper power, as $\Omega_{0} \propto \sqrt{P}$.

With the laser-induced switchable coupling we now discuss how to implement the universal quantum gates by non-adiabatically manipulations. For generality, we consider here $n(\geq 2)$ potential wells (the adjacent wells are separated by an uniform distance $d$ ), as shown in Fig. 3. Each potential well confines two ions, e.g., $Q_{n}$ and $A_{n}$. The ion $Q_{n}$ (e.g., a ${ }^{9} \mathrm{Be}^{+}[9]$ ) is used to encode the qubits by its long-lived internal states $|\downarrow\rangle$ and $|\uparrow\rangle$. While, the auxiliary ion $A_{n}$ (e.g., a ${ }^{40} \mathrm{Ca}^{+}[10]$ ) is used for two purposes: (i) cooling the $\mathrm{CM}\left|\psi_{\mathrm{cm}}\right\rangle_{n}$ of the two ions $Q_{n}$ and $A_{n}$ confined in the same trap, and (ii) implementing the switchable coupling $\hat{U}_{n, n+1}(g t)$ between the CMs of the ions trapped in adjacent potential wells.

In the same potential well the frequency of ions' breath mode (BM) is significantly larger than that of the CM, so that we can treat them separately [18, 19]. Assuming the $n$ potential wells have any different depths such that the CM-CM, BM-BM, and BM-CM couplings between the different wells are naturally cut off unless the laser-assisted coupling $\hat{U}_{n, n+1}(g t)$ is applied. By selecting the proper sideband laser pulses the quantum information (QI) between the internal states and $\mathrm{CM}$ can be transferred, while the BM does not work (since it has different frequency 

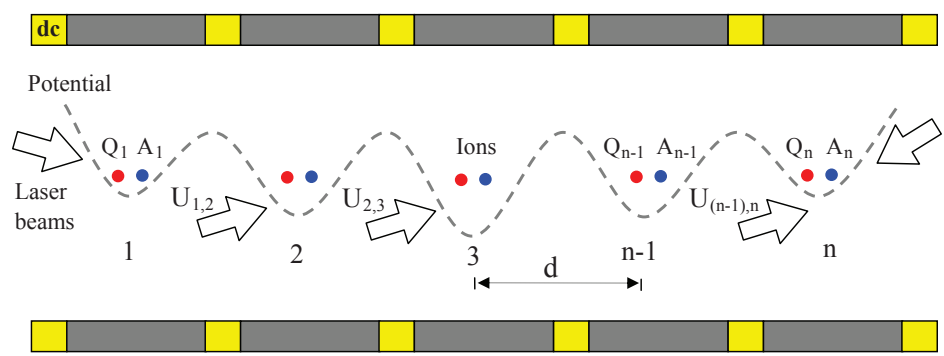

FIG. 3: (Color online) Sketch for implementing quantum logic operations with $n(\geq 2)$ potential wells. The distance between the adjacent wells is $d$. Each potential well confines two ions: the red ion (e.g. the ion $Q_{n}$ ) is used to encode the qubit, and the blue ion (e.g., the ion $A_{n}$ ) is used to cool the ions' center-of-mass mode $(\mathrm{CM})$ and implement the switchable coupling between the ionic vibrations in the adjacent potential wells.

respect to the CM) [20]. Therefore, one can use only CM as the desirable data bus to realize the QI transformation between the qubit trapped in the different potential wells. Since we are considering two different ions (i.e., ${ }^{9} \mathrm{Be}^{+}$and ${ }^{40} \mathrm{Ca}^{+}$) in each potential well, the laser individually driving is unnecessary. The laser is required only to be focused onto a waist smaller than $d \sim 40 \mu \mathrm{m}$ (the distance between the adjacent potential wells [9]), in principle.

It has been well-known that the single-qubit operations can be exactly implemented by driving various resonant Raman transitions [21, 22]. The present purpose is to implement the two-qubit controlled-NOT (CNOT) gate [2]

$$
\hat{C}_{1, n}=\left(\begin{array}{cccc}
1 & 0 & 0 & 0 \\
0 & 1 & 0 & 0 \\
0 & 0 & 0 & 1 \\
0 & 0 & 1 & 0
\end{array}\right)
$$

between the qubits (e.g., the ions $Q_{1}$ and $Q_{n}$ ) trapped in the different potential wells. For simplicity, we suppose that the CMs are initially prepared in the vacuum states $\prod_{j=1}^{n}\left|0_{\mathrm{cm}}\right\rangle_{j}$. Then, the desirable CNOT gate $\hat{C}_{1, n}$ could be realized by the following operational sequence:

$$
\hat{C}_{1, n}=\hat{V}_{1} \hat{M}_{n, 1} \hat{S}_{n} \hat{M}_{1, n} \hat{V}_{1}
$$

Here, $\hat{V}_{1}$ represents an operation $\left|0_{\mathrm{cm}}, \uparrow\right\rangle_{1} \longrightarrow \exp [i(1-n) \pi / 2]\left|1_{\mathrm{cm}}, \downarrow\right\rangle_{1}$ applied on ion $Q_{1}$ for transferring the QI from its internal states to the CM in potential well 1. Experimentally, the operation $\hat{V}_{1}$ can be realized by applying a red-sideband laser pulse to the ion, see, e.g., Refs. [20, 
21]. The operation $\hat{M}_{1, n}=\prod_{j=1}^{n-1} \hat{U}_{(n-j),(n-j+1)}(\pi / 2)$ represents $n-1$ resonant pulses applied sequentially on the auxiliary ions $A_{2}, A_{3}, \cdots$, and $A_{n}$ to couple the CMs in the separated potential wells one by one. Physically, the operation $\hat{M}_{1, n}$ transfers the QI stored in the CM of potential well 1 to that in the distant well $n$. Similarly, $\hat{M}_{n, 1}=\prod_{j=1}^{n-1} \hat{U}_{j,(j+1)}(\pi / 2)$ transfers the QI from the potential well $n$ back to the well 1 . Finally, $\hat{S}_{n}$, a single-ion CNOT gate between the CM and internal states of the ion $Q_{n}$, can be implemented by applying three sequential laser pulses to the ion (as the demonstrations in the experiment [21]).

The proposed CNOT gate $\hat{C}_{1, n}$ could be implemented within a short operational time. First, the durations of operation $\hat{V}_{1}$ and gate $\hat{S}_{n}$ by using the Raman schemes of experiment [21] are estimated as $t_{\mathrm{v}} \approx 8 \mu \mathrm{s}$ and $t_{\mathrm{s}} \approx 50 \mu \mathrm{s}$, respectively. Second, for the typical parameters [9, 10]: $d \approx 40 \mu \mathrm{m}$ (the distance between the adjacent potential wells) and $\nu_{j} \approx 5 \mathrm{MHz}$ (the vibrational frequencies of the CMs), the coupling strength between the adjacent CMs is calculated as $g_{\mathrm{cm}} \approx$ $35 \mathrm{kHz}$, and thus the duration of the operation $\hat{U}_{j,(j+1)}(\pi / 2)$ is $t_{\mathrm{u}} \approx 45 \mu \mathrm{s}$. Obviously, all these durations (i.e., $t_{\mathrm{v}}, t_{\mathrm{s}}$, and $t_{\mathrm{u}}$ ) are significantly shorter than the coherent times (which is about $1 \mathrm{~ms}$ ) of the CMs [9, 10, 20].

Note that the laser coolings of CMs performed by using the auxiliary ions do not affect the qubits. Therefore, during the operation $V_{1}, \hat{S}_{n}$, or $\hat{U}_{j,(j+1)}$ applied on the ions $1, n$, or $j$ and $j+1$, the other ions can be re-cooled. This implies that the practical limit for the implementation of CNOT gate $\hat{C}_{1, n}$ is the decoherence from the states $|\downarrow\rangle$ and $|\uparrow\rangle$, i.e., the qubit. Fortunately, the coherent times between the selected internal states $|\downarrow\rangle$ and $|\uparrow\rangle$ are relative long, e.g, up to $\sim 10 \mathrm{~s}$ [23]. This allows us to implement the desirable gates between a large number of trapped ions, e.g., $n \sim 20$. Indeed, the total duration $t_{\text {total }}=2\left[t_{\mathrm{v}}+(n-1) t_{\mathrm{u}}\right]+t_{\mathrm{s}} \approx 1.8 \mathrm{~ms}$ for generating the CNOT gate $\hat{C}_{1,20}$ is far shorter than the coherent time of the states $|\downarrow\rangle$ and $|\uparrow\rangle$. Consequently, entangling a large number of ions (by using a series of single-qubit operations and CNOT gates) may be possible.

In conclusion, we have proposed an alternative approach to implement the universal quantum gates between the separately trapped ions. Instead of the previous adiabatic operations of potential wells, here the laser beams are utilized to non-adiabatically manipulate the ion-ion couplings. Consequently, the desirable quantum gates between the ions could be conveniently implemented by using only a series of laser pulses. A key feature of our proposal is that it can be generalized to the scaling ion-traps. With the experimental parameters, e.g., the quadrupole transition Rabi frequency, the vibrational frequencies of the trapped ions, and the distance between the potential 
wells, we have analyzed the feasibility of the approach in detail.

Acknowledgements: This work was partly supported by the National Natural Science Foundation of China Grants No. 11147116, 10874142 and No. 90921010, the Major State Basic Research Development Program of China Grant No. 2010CB923104 (through Program No. 973), and the open project of State Key Laboratory of Functional Materials for Informatics.

[1] Ladd T D et al 2010 Nature $\mathbf{4 6 4} 45$

[2] Cirac J I and Zoller P 1995 Phys. Rev. Lett. 744091

[3] Mølmer K and Sørensen A 1999 Phys. Rev. Lett. 821835

[4] Häffner H, Roos C F and Blatt R 2008 Phys. Rep. 469155

[5] Monz T et al 2011 Phys. Rev. Lett. 106130506

[6] Kielpinski D, Monroe C and Wineland D J 2002 Nature 417709

[7] Home J P et al 2009 Science 3251227

[8] Cirac J I and Zoller P 2000 Nature 404579

[9] Brown K R et al 2011 Nature 471196

[10] Harlander M et al 2011 Nature 471200

[11] Zhang M and Wei L F 2011 Phys. Rev. A 83064301

[12] Leibfried D et al 2003 Rev. Mod. Phys. 75281

[13] Bermudez A, Schätz T and Porras D 2011 Phys. Rev. Lett. 107150501

[14] Roos C et al 1999 Phys. Rev. Lett. 834713

[15] Liu Q et al 2011 Chin.Phys. Lett. 28013201

[16] Shu H L et al 2007 Chin.Phys. Lett. 241217

[17] Poschinge U G et al 2009 J. Phys. B 42154013

[18] Steane A 1997 Appl. Phys. B. 64623

[19] James D F V 1998 Appl. Phys. B. 66181

[20] Schmidt-Kaler F et al 2003 Nature 422408

[21] Monroe C et al 1995 Phys. Rev. Lett. 754714

[22] Meekhof D M et al 1996 Phys. Rev. Lett. 761796 
[23] Langer C et al 2005 Phys. Rev. Lett. 95060502 